
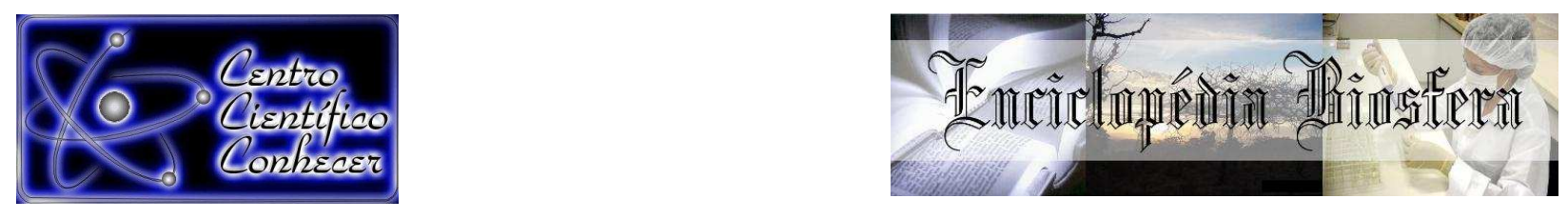

\title{
LIGAÇÃO DA ARTÉRIA CORONÁRIA ESQUERDA: UMA TÉCNICA DE ESTUDO DAS ALTERAÇÕES ISQUÊMICAS DO MIOCÁRDIO
}

Fernanda Almeida Rodrigues ${ }^{1}$, Vanessa de Sousa Cruz Pimenta ${ }^{2}$, Eugênio Gonçalves de Araújo ${ }^{3}$

1 Doutoranda do Programa de Pós-Graduação em Ciência Animal da Escola de

Veterinária e Zootecnia da Universidade Federal de Goiás

(fernanda_rodrigues90@hotmail.com)

Goiás-Brasil

2 Pós doutoranda, Laboratório Multiusuário de Cultivo Celular da Universidade

Federal de Goiás

3 Professor Doutor do Setor de Patologia Veterinária da Universidade Federal de Goiás

Recebido em: 08/04/2016 - Aprovado em: 30/05/2016 - Publicado em: 20/06/2016 DOI: 10.18677/Enciclopedia_Biosfera_2016_062

\begin{abstract}
RESUMO
Doenças cardiovasculares são situações correlacionadas com o mal funcionamento do bombeamento do sangue. As arritmias e infarto agudo do miocárdio estão entre as principais doenças cardiovasculares. Os hábitos de vida irregulares e doenças endócrinas são os fatores de risco mais comuns. Para estudar os mecanismos de ação e tratamentos para prevenir o infarto, utilizam-se algumas técnicas como a oclusão hidráulica ou constritor anelar e embolização da artéria coronária, em animais de experimento. A ligação da artéria coronária esquerda leva à diminuição do volume sistólico, débito cardíaco, pressão sistólica dentro do ventrículo esquerdo e aumento da pressão diastólica no final do ventrículo esquerdo. Os estudos de indução do infarto agudo do miocárdio, em modelos experimentais, são ideais para avaliar a intensidade das alterações fisiopatológicas que ocorrem em seres humanos.
\end{abstract}

PALAVRAS-CHAVE: Infarto do miocárdio, insuficiência cardíaca, isquemia.

\section{THE LEFT CORONARY ARTERY: A STUDY OF ISCHEMIC MYOCARDIAL ALTERATIONS}

\begin{abstract}
The cardiovascular diseases are correlated situations with the badly function of the blood plumping. Arrhythmias and acute myocardial infarction are among the main cardiovascular diseases. The irregular lifestyle habits and endocrine diseases are the most common risk factors. Some of technics are used to study the mechanism of action and the treatments to prevent heart attack, such as hydraulic occlusion or constrictor ring and coronary artery embolization in experimental animals. The connection of the left coronary artery leads to decreased stroke volume, cardiac output, and into the left ventricle and the diastolic pressure reflect an increase in the
\end{abstract}


final left ventricle. The studies of induction of acute myocardial infarction in experimental models are ideal to assess the intensity changes of psychopathological occurrence in humans.

KEYWORDS: Myocardial infarction, cardiac insufficiency, ischaemia

\section{INTRODUÇÃO}

O coração é um órgão muscular localizado na caixa torácica, mantido em posição vertical pelas veias cavas inferiores e superiores e horizontalmente pelas veias pulmonares. Seu ápice é elevado de encontro ao tórax durante a diástole (FERREIRA \& POVOA, 1999; SILVA et al., 2013). Internamente, o coração é dividido em quatro câmaras, separadas em átrios e ventrículos, por válvulas septadas, em direito e esquerdo (SPOSITO et al., 2007). Para desempenhar o papel de bomba propulsora de sangue oxigenado para todo organismo, o coração normal deve ejetar e receber a quantidade de sangue necessária para produzir o débito cardíaco (FERREIRA \& POVOA, 1999).

Doenças cardiovasculares são situações correlacionadas com o mal funcionamento do bombeamento do sangue. Estão entre as principais causas de morte e são consideradas como "doença dos homens de meia idade" (SOLIMENE, 2015). Em todo o mundo, essas doenças destacam-se de maneira expressiva nos índices de gastos com saúde pública e mortalidade (BRASIL, 2006). No ano de 2009, o número de internações por doenças cardiovasculares registrado no Sistema Único de Saúde Brasileiro foi de 91.970 casos. Os tratamentos são procedimentos de alta complexidade e com custo elevado por paciente. O recurso gasto pelo governo brasileiro foi da ordem de $R \$ 165.461 .644,33$, impactando diretamente no orçamento dos órgãos de Saúde. Segundo o Ministério da Saúde, em 2011, 60.158 dos brasileiros acometidos vieram a óbito; deste total 15.023 eram do sexo masculino e a prevalência da idade variou entre 60 a 69 anos (BRASIL, 2014).

Hábitos de vida irregulares e doenças endócrinas são os fatores de risco mais comuns. As arritmias e infarto agudo do miocárdio estão entre as principais doenças cardiovasculares (TABTABAl et al., 2015). O infarto agudo do miocárdio é uma lesão irreversível, resultante da obliteração dos vasos sanguíneos, responsáveis pela irrigação do coração (CHANG et al., 2013), frequentemente como consequência de placas de ateroma (MULDER et al., 2014).

Em animais de uso experimental, é possível a análise de substâncias farmacológicas, regiões limitadas do infarto, dilatação ventricular, formação de aneurisma, desempenho do ventrículo e expansão do infarto. Os procedimentos modelos de isquemia e reperfusão, infarto agudo do miocárdio e ligação da artéria coronária esquerda podem ser induzidos (VERDOUW et al., 1998).

Diante do exposto, esta revisão de literatura tem o propósito de apresentar a técnica de ligação da artéria coronária esquerda como um método de indução do infarto agudo de miocárdio em animais de experimentação, visando o desenvolvimento de novas terapias a serem utilizadas no paciente humano para minimizar a necrose do músculo cardíaco e reduzir o índice de mortalidade mundial.

\section{SISTEMA CARDIOVASCULAR}

Nos organismos multicelulares não há comunicação das células com o meio ambiente, o que dificulta a absorção de nutrientes e a eliminação de metabólitos tóxicos. No entanto, estes organismos apresentam um sistema fechado que executa estas funções, denominado de sistema circulatório (Figura 1) ou cardiovascular (GARTNER \& HIATT, 1993; DÂNGELO \& FATTINI, 2000). 


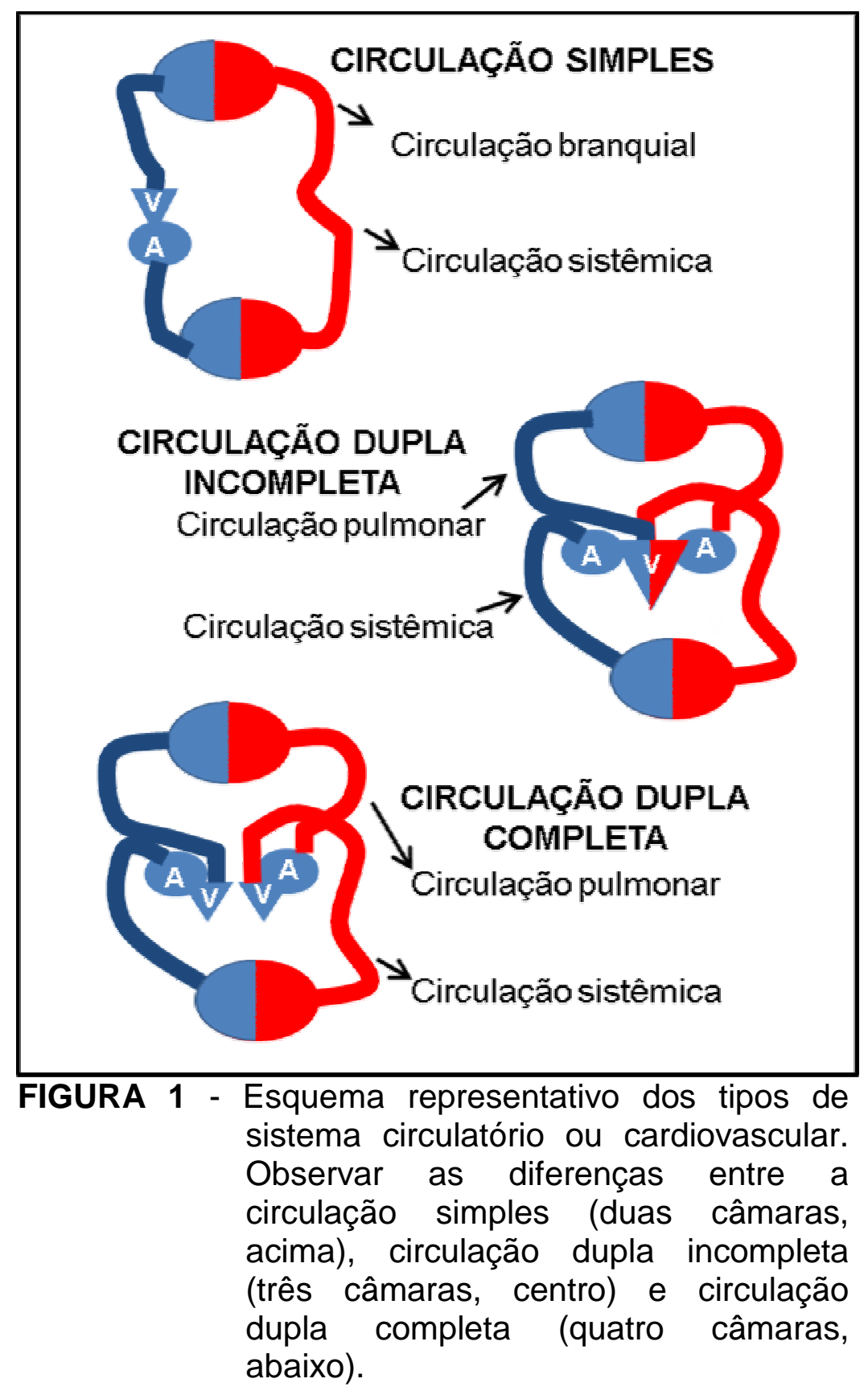

Nos peixes, o coração apresenta-se em forma de "S". O sangue vindo das veias entra pelo seio venoso, é direcionado para o átrio, ventrículo. Este bombeia o sangue pobre em oxigênio para as brânquias, nelas ocorre a troca gasosa, em seguida este sangue é drenado para aorta dorsal e distribuído pelo corpo do animal. Nos répteis, o coração se apresenta dividido em três câmaras: átrio direito, átrio esquerdo e ventrículo. O sangue rico em oxigênio, vindo do pulmão, chega pelo átrio esquerdo. O baixo turbilhonamento, a parede esponjosa do ventrículo, a septação do bulbo arterial e a válvula espiral o direcionam do átrio esquerdo para o corpo, pelo arco arterial. Já o sangue vindo do corpo com dióxido de carbono, chega pelo átrio direito sem se misturar ao sangue oxigenado, devido a septação, sendo direcionado para a artéria pulmocutânea e levado aos pulmões e pele (SPOSITO et al., 2007).

Nos humanos e demais mamíferos, a circulação é fechada. O sangue vindo dos pulmões, rico em oxigênio, chega ao coração pelo lado esquerdo e é 
drenado pelo tronco sistêmico para todo o corpo, denominada de circulação sistêmica. O lado direito drena o sangue pouco oxigenado para os pulmões através do tronco pulmonar, o que consiste na circulação pulmonar. O sangue é impedido de retornar devido à propulsão feita pelas válvulas, cordas e músculos (SPOSITO et al., 2007).

Considerando a distribuição do fluxo sanguíneo, o sistema cardiovascular é subdivido em circuito pulmonar e circuito sistêmico. O circuito pulmonar leva o sangue da câmara direita do coração ao pulmão para ser oxigenado, retornando-o à câmara esquerda (SPOSITO et al., 2007). O circuito sistêmico inicia-se nesta câmara, impulsionando o sangue oxigenado para todo o organismo e em seguida retornando-o à câmara direita, completando o ciclo. Basicamente, este sistema é constituído de sangue (fluído sanguíneo), coração (bomba circulatória), artérias (rede de distribuição), veias (rede de coleta) e microcirculação (sistema de oxigenação e troca metabólica) (GARTNER \& HIATT, 1993).

\section{SANGUE}

Com uma coloração vermelho escura, o sangue é um tecido conjuntivo constituído por hemácias, leucócitos, plasma, plaquetas e fatores de coagulação. As hemácias são glóbulos vermelhos, responsáveis pelas trocas gasosas. São anucleadas e possuem diâmetro variando entre $6 \mu \mathrm{m}$ e $8 \mu \mathrm{m}$. Os leucócitos são glóbulos brancos, com função de resposta imunológica. Possuem núcleos, na grande maioria, grânulos e diâmetro variando de $8 \mu \mathrm{m}$ a $14 \mu \mathrm{m}$. O plasma é o componente líquido responsável por $55 \%$ do volume sanguíneo. Em sua composição encontram-se sais e íons (cálcio, sódio, potássio e bicarbonato), moléculas grandes (albumina, globulina e fibrinogênio) e compostos orgânicos (aminoácidos, lipídios, vitaminas, hormônios e cofatores) (GARTNER \& HIATT, 1993).

\section{CORAÇÃO}

Em humanos, o coração localiza-se lateralmente aos pulmões, anteriormente ao esterno, posteriormente à coluna vertebral e inferiormente ao diafragma (SILVA, 2013). O ápice está situado entre o quarto e o quinto espaço intercostal, em contato com a caixa torácica (FERREIRA \& POVOA, 1999). A relação entre o peso do coração e o peso corporal é de aproximadamente $5 \mathrm{~g} / \mathrm{kg}$ em humanos e suínos. Já em cães esta relação é duas vezes maior (VERDUW et al., 1998).

Para que o sangue chegue às células do organismo é necessário que aconteça o bombeamento, por um órgão de musculatura estriada transversais com células de núcleo central e discos intercalares, denominado coração (JUNQUEIRA \& CARNEIRO, 1995). Este órgão é constituído por três camadas: o epicárdio ou pericárdio, o miocárdio e o endocárdio.

O epicárdio é a camada mais externa, recoberta por um mesotélio pavimentoso simples, tecido conjuntivo fibroelástico e tecido adiposo, contendo vasos e nervos coronarianos. A camada intermediária, denominada de miocárdio, é composta por fibras musculares cardíacas ligadas ao tecido conjuntivo colágeno. Internamente o coração é revestido por um endotélio pavimentoso simples e um tecido conjuntivo fibroelástico subendotelial (GARTNER \& HIATT, 1993).

$\mathrm{Na}$ morfologia interna, o coração apresenta-se com paredes abertas, formando câmaras direita e esquerda, que são subdividas pelos septos: átrioventricular - sentido horizontal e inter-atrial e inter-ventricular - sentido vertical. Entre 
os átrios ventriculares existem orifícios, óstios, que permitem a passagem do sangue, providos de válvulas que impedem esta passagem livremente, denominadas de válvulas átrio-ventriculares. As válvulas são formadas por tecido conjuntivo denso e recobertas pelo endocárdio. A chegada de sangue no coração ocorre por meio das veias e a saída por meio das artérias (ANDRADE, 2006; SPOSITO et al, 2007).

A válvula aórtica separa a artéria aorta do ventrículo esquerdo. Estruturalmente apresenta três cúspides, que durante a sístole voltam-se para o seio, abrindo a passagem do fluxo sanguíneo e durante a diástole estendem-se, impedindo a passagem deste fluxo (LOOI et al., 2015). O fornecimento de oxigênio e nutrientes às células, a excreção de toxinas provenientes do metabolismo celular, a condução de hormônios e células de defesa para o todo organismo são funções que dependem da interconecção de vasos sanguíneos ao coração (SILVA et al., 2013).

\section{VASOS SANGUÍNEOS}

Os vasos sanguíneos, veias e artérias, são microscopicamente constituídos pelas túnicas íntima, média e adventícia. A túnica íntima ou interna é representada por um epitélio conjuntivo frouxo de células endoteliais, recoberto por um limitante elástico interno, tubular e perfurado. A túnica média é formada por fibras musculares lisas, dispostas circularmente e agregadas a fibras elásticas e fibras reticulares, como colágenos tipo III e proteoglicanas. A túnica adventícia localiza-se mais externamente e consiste de tecido conjuntivo, com fibras colágenas tipo I e elásticas (JUNQUEIRA \& CARNEIRO, 1995).

A diferenciação entre veias e artérias já se apresenta evidente no aspecto macroscópico. As veias são de coloração azul-escura, paredes finas e diâmetro maior do que das artérias, com circulação centrípeta e válvulas internas para evitar o retorno sanguíneo. As artérias apresentam-se como tubos cilíndricos, elásticos, de coloração branca-amarelada e circulação centrífuga (DÂNGELO \& FATTINI, 2000).

As artérias ao serem obstruídas há a diminuição do fluxo sanguíneo de forma parcial ou total, então acontece o processo de isquemia. A interrupção do oxigênio leva à disfunção das mitocôndrias, que reduzem a produção de adenosina trifosfato, diminuindo a contratibilidade miocárdica. A glicogenólise é ativada, o nível de nicotinamida adenina dinucleotidio reduzido, o de dinucleotído flavina e adenina aumentado e o pH diminui. $\mathrm{O}$ meio intracelular se acidifica ainda mais com 0 aumento da concentração dos íons cálcio, diminuição de sódio e de potássio. Há o aumento dos teores de metabolitos nas células, como acúmulo de metabólitos a partir da glicogenólise, como lactato, a partir do uso de fosfocreatininas, como creatinina e fosfatos orgânicos (FABER, 1981), levando ao acúmulo de água dentro das células. Após a desobstrução das artérias, o fluxo sanguíneo pode ser restaurado. A reperfusão pode produzir espécies reativas de oxigênio, aumentando a quantidade de íons cálcio dentro das mitocôndrias, levando à necrose ou apoptose. Qualquer alteração que comprometa o funcionamento normal dos vasos e artérias do coração pode desencadear doenças cardiovasculares (BOPASSA, 2012).

\section{DOENÇAS CARDIOVASCULARES}

As doenças cardiovasculares comprometem o funcionamento normal de vasos arteriais e do coração. Com o passar dos anos, os hábitos de vida da população mundial foram se modificando com dietas e atividades físicas inadequadas e insuficientes. A descoberta de novas tecnologias de diagnóstico (MS/SAS) contribuíram para o desencadeamento e evidenciamento dos fatores de 
risco e do desenvolvimento das cardiopatias. Dentre os fatores de risco (MS/SAS), existem os não modificáveis e os modificáveis por mudanças de hábitos de vida ou medicamentos (Quadro 1) (SIMÃO et al., 2002; BRASIL, 2012).

QUADRO 1 - Fatores de risco não modificáveis e os modificáveis que podem desencadear doenças cardiovasculares.

\begin{tabular}{|c|c|}
\hline & FATORES DE RISCO \\
\hline Não & História familiar de doença arterial cardiovascular prematura \\
\hline Modificáveis & $\begin{array}{c}\text { (familiar } 1^{\circ} \text {. grau sexo masculino }<55 \text { anos e sexo feminino }<65 \\
\text { anos); }\end{array}$ \\
\hline \multirow{10}{*}{ Modificáveis } & Homem $>45$ anos e mulher $>55$ anos \\
\hline & Tabagismo \\
\hline & Hipercolesterolemia (LDL-c elevado) \\
\hline & Hipertensão arterial sistêmica \\
\hline & Diabete melito \\
\hline & Obesidade (IMC > $\left.30 \mathrm{~kg} / \mathrm{m}^{2}\right)$ \\
\hline & Gordura abdominal \\
\hline & Sedentarismo \\
\hline & obre em frutas e vegetais \\
\hline & stresse psicossocial \\
\hline
\end{tabular}

Fonte: Adaptado por SIMÃO et al. 2002

Doenças como arritmias cardíacas, anginas, isquemias e infarto agudo do miocárdio são provenientes do acúmulo de fatores de risco comportamentais, levando a formação de placas ateroscleróticas nas paredes das artérias, obstruindo a passagem do fluxo sanguíneo, afetando diretamente o coração (RADILSON, 2011).

As placas ateroscleróticas são formadas quando o endotélio vascular é agredido pelas lipoproteínas aterogênicas, estas são retidas no espaço subendotelial e oxidadas. Os monócitos migram até estas lipoproteínas oxidadas, se diferenciam em macrófagos e captam estas moléculas lipoproteicas, formando células espumosas. Mediadores da resposta inflamatória migram até estas células, produzem citocinas, fatores de crescimento, matriz extracelular e formam a capa fibrosa da placa aterosclerótica (SPOSITO et al., 2007). Em animais saudáveis de uso experimental, pode-se induzir isquemias e reperfusões, infartos agudo do miocárdio, como a ligação da artéria coronária esquerda (VERDOUW et al., 1998).

\section{INFARTO AGUDO DO MIOCÁRDIO}

É descrito como a morte de cardiomiócitos (necrose) causada por uma isquemia (PESARO, 2004)' insuficiência de oxigênio e de nutrientes do musculo cardíaco, diante da alteração da obstrução de uma artéria coronária por período prolongado, podendo ser por coágulos sobre as placas ateroscleróticas (PESARO et al., 2004; WHITE \& CHEW, 2008).

Os fatores de risco indutores do infarto são os mesmos das demais doenças cardiovasculares, como predisposição genética, idade, sexo, fumo, obesidade, diabetes e hipertensão. Porém, são considerados fatores agravantes a elevação dos índices de colesterol e gordura saturada elevada (THYGESEN et al., 2012; CHANG et al., 2013). Em 2012, os tipos de infarto agudo do miocárdio foram classificados com base nas diferenças patológicas, clínicas e no diagnóstico. Esta classificação está descrita no Quadro 2. 
Com a finalidade de estudar os mecanismos de ação e tratamentos para prevenir o infarto, utiliza-se a reprodutibilidade do infarto agudo do miocárdio em animais de experimentação, relacionada diretamente com a determinação da duração, extensão e reperfusão do fluxo sanguíneo. Algumas técnicas como a oclusão hidráulica ou constritor anelar e embolização da artéria coronária são utilizadas, porém a ligação da artéria coronária é a mais empregada (KLOCKE et al., 2007).

Em modelo experimental suíno, a artéria coronária pode ser ocluída de forma parcial ou total, por meio da implantação de um oclusor em forma de "U" ou anelar. Após a instalação dos oclusores, mede-se o fluxo sanguíneo. Para confirmar o infarto, implanta-se uma sonda na artéria coronária e verifica-se a ausência do fluxo sanguíneo. Já a técnica da embolização da artéria coronária foi desenvolvida em cães como modelo experimental. Consiste na instalação intra-arterial de microesferas de agarose ou de poliestireno, expandidas com trombina e sangue autógeno com fibrinogênio. Há um aumento da pressão na diástole e da artéria pulmonar. A utilização é limitada por não ser possível controlar a localização exata e o comprimento da oclusão da artéria coronária (KLOCKE et al., 2007).

A técnica da ligação da artéria coronária foi inicialmente desenvolvida utilizando-se o cão como modelo experimental. Porém, a variabilidade anatômica (JOHNS \& OLSON, 1954; KLOCKE et al., 2007), o custo e a pressão social fizeram com que outros animais, como ratos, macacos e camundongos, fossem utilizados em laboratório para este fim (VERDOUW et al., 1998; ZORNOFF et al.; 2009, XU et al., 2014).

QUADRO 2 - Classificação do infarto agudo do miocárdio baseados em diferenças patológicas, clínicas e no diagnóstico.

\begin{tabular}{|c|c|c|}
\hline \multicolumn{2}{|c|}{$\begin{array}{l}\text { TIPOS DE INFARTO AGUDO DO } \\
\text { MIOCÁRDIO }\end{array}$} & FATORES DE RISCO \\
\hline Tipo 1 & Espontâneo & $\begin{array}{l}\text { Placa aterosclerótica se rompe, o fluxo } \\
\text { intraluminal diminui, os miócitos } \\
\text { necrosam, o paciente pode ou ter } \\
\text { doença coronária arterial. }\end{array}$ \\
\hline Tipo2 & $\begin{array}{l}\text { Secundário a um } \\
\text { desequilíbrio da isquemia }\end{array}$ & $\begin{array}{l}\text { Em doenças coronarianas com necrose } \\
\text { há um desequilíbrio entre a oferta e a } \\
\text { demanda de oxigênio. }\end{array}$ \\
\hline Tipo 3 & $\begin{array}{l}\text { Resulta em morte quando } \\
\text { os valores dos } \\
\text { biomarcadores não estão } \\
\text { disponíveis }\end{array}$ & $\begin{array}{l}\text { Morte cardíaca sugere sintomas de } \\
\text { isquemias e alterações do } \\
\text { eletrocardiograma. A morte ocorre } \\
\text { antes da coleta de sangue e análise dos } \\
\text { biomarcadores. }\end{array}$ \\
\hline Tipo $4^{\underline{a}}$ & $\begin{array}{l}\text { Relacionado com } \\
\text { intervenção coronária } \\
\text { percutânea }\end{array}$ & $\begin{array}{l}\text { Valores de tropomiosina cardíaca estão } \\
\text { elevados, novas lesões isquêmicas no } \\
\text { eletrocardiograma, persistência de } \\
\text { baixo fluxo sanguíneo. }\end{array}$ \\
\hline $\begin{array}{l}\text { Tipo } \\
4 \mathrm{~b}\end{array}$ & $\begin{array}{l}\text { Relacionado com } \\
\text { estenose trômbica }\end{array}$ & $\begin{array}{l}\text { Trombose é detectada na angiografia } \\
\text { coronária e aumento e/ou queda dos } \\
\text { biomarcadores. }\end{array}$ \\
\hline Tipo 5 & $\begin{array}{l}\text { Relacionado a cirurgia de } \\
\text { revascularização }\end{array}$ & $\begin{array}{l}\text { Elevação dos biomarcadores cardíacos, } \\
\text { oclusão coronariana ativa, movimento } \\
\text { anormal da parede do miocárdio. }\end{array}$ \\
\hline
\end{tabular}

Fonte: Adaptado de THYGESEN et al, 2012 


\section{LIGAÇÃO DA ARTÉRIA CORONÁRIA ESQUERDA}

A artéria coronária esquerda, em humanos, possui trajeto horizontal leve ou caudo-cranial. A origem é no seio aórtico esquerdo, passando atrás do tronco pulmonar. Divide-se em artéria descendente anterior e artéria circunflexa (ANDRADE, 2006).

As remodelações fisiopatológicas do coração após a ocorrência do infarto agudo do miocárdio podem ser reproduzidas e avaliadas por meio da técnica de ligação da artéria coronária esquerda (KLOCHE et al., 2007; ZORNOFF et al., 2009). Em uma análise da anatomia das artérias coronárias dos roedores, verificou-se que nos ratos e camundongos esta artéria apresenta-se com um tronco grande e sem muitas ramificações. Nos hamsters e cobaias a oclusão arterial é dificultada, devido a um ramo circunflexo se originar próximo ao início da artéria coronária esquerda (JOHNS \& OLSON, 1954; HOUSER et al., 2012). Experimentos de ligação da artéria coronária esquerda realizados em ratos permitem a análise de substâncias farmacológicas, regiões limitadas do infarto, dilatação ventricular, formação de aneurisma, performance do ventrículo, expansão do infarto e os sinais e sintomas pós-cirúrgicos (COOPER et al., 1995).

A obstrução de vasos coronários desencadeia, de imediato, a formação do processo de angiogênese. Nos humanos, esta formação de circulação colateral é lenta (VERDOUW et al., 1998). Nos modelos experimentais com cães, ratos e camundongos, esta mesma característica está presente, o que permite o estudo do infarto agudo do miocárdio (VERDOUW et al., 1998; ZORNOFF et al., 2009).

No caso da ligação da artéria coronária direita, ocorre a indução do infarto em $85 \%$ da parede livre e do septo anterior do ventrículo direito. No entanto há uma taxa de mortalidade de $15 \%$ imediatamente após o infarto, devido a alterações acentuadas nos padrões hemodinâmicos (SAMSAMSHARITA \& MOVAHEDB, 2005).

O tamanho da área do infarto, quando se utiliza a ligação da artéria coronária esquerda, pode ser mensurado por uma média de cortes transversais, já que o infarto acomete mais a região apical do que a região da base do ventrículo esquerdo. Após a oclusão coronária ocorre a remodelação tecidual, com ativação de enzimas proteinases, as metaloproteases, que irão degradar o colágeno fibrilar. Infiltrado de linfócitos, monócitos e neutrófilos desencadearão o processo de necrose em 24 horas. No terceiro dia, os fibroblastos modificados sintetizam colágeno I e III, que se depositam na região periférica do infarto, iniciando o processo de cicatrização (ZORNOFF et al., 2009).

O cálculo em porcentagem do infarto do epicárdio e do endocárdio pode ser feito por meio do somatório do comprimento da cicatriz do epicárdio, dividido pela somatória da cicatriz circunferencial do epicárdio multiplicado por 100. Para o cálculo da porcentagem total do infarto, faz-se uma média entre os infartos epicárdicos e endocárdicos (HOEH et al., 2012). Uma outra maneira de determinar a porcentagem da área infartada em ratos é a imersão do coração em meio próprio para congelamento à temperatura de $-20^{\circ} \mathrm{C}$. As secções devem ser realizadas entre o ápice e a válvula mitral. O cálculo será: Áreas do infarto $(\%)=$ (circunferência da área infartada do ventrículo esquerdo / circunferência total do ventrículo esquerdo) $X$ 100 (COOPER et al., 1995).

\section{PROTOCOLO CIRÚRGICO}

Inicialmente, os ratos são anestesiados até a perda dos reflexos, com isofluorano a $5 \%$ e oxigênio, num fluxo de $0,4 \mathrm{~L} / \mathrm{min}$. A manutenção da anestesia é 
feita com a utilização de $2 \%$ de isofluorano em $100 \%$ de oxigênio, com o fluxo mantido em 0,4 L/min (XU et al., 2014). As anestesias intraperitoneais ou intramusculares podem ser feitas pela administração de barbitúricos ou associação de cloridrato de cetamina $(50 \mathrm{mg} / \mathrm{kg})$ e cloridrato de xilidino $(1 \mathrm{mg} / \mathrm{kg})($ ZORNOFF et al., 2009).

Para a realização da toracotomia, posiciona-se o rato em decúbito lateral esquerdo, faz-se uma incisão longitudinal oblíqua de aproximadamente $1 \mathrm{~cm}$ (XU et al., 2014), entre o quarto e o quinto espaços intercostais, visualizando coração (JOHNS \& OLSON, 1954; AGNOLETTI et al., 2006). Realiza-se, então, uma leve compressão lateral no tórax e exterioriza-se o coração. A artéria coronária esquerda será evidenciada e estará exposta. Em seguida, passa-se o fio de sutura 5-0 em torno desta a artéria, a $2 \mathrm{~mm}$ de sua origem, entre a borda do átrio esquerdo e 0 sulco da artéria pulmonar (Figura 10C e 10D) (ZORNOFF et al., 2009; LUO et al., 2015). Efetiva-se dois nós com pressão, para ocluir a passagem do fluxo sanguíneo, porem com o cuidado de não romper a artéria (XU et al., 2014). Ao utilizarem-se procedimentos de isquemia e reperfusão após a exposição do coração intratorácico, deve ser realizada a ligação da artéria coronária com oclusão total, porém reversível, promovendo o processo de isquemia por um período de tempo pré-definido. Passado este tempo, retira-se a sutura e o sangue retoma o seu fluxo contínuo, por tempo também pré-definido (SANTIAGO et al., 2009). Finaliza-se com a recolocação do coração na cavidade torácica, expansão dos pulmões e fechamento do tórax (ZORNOFF et al., 2009; LUO et al., 2015).

Durante o pós-operatório, os animais são avaliados em relação a depleção da função cardíaca, hipertrofia do ventrículo, aumento da pressão diastólica e diminuição da sistólica e aumento da angiotensia II, nos dias que se seguem e as medicações administradas conforme os protocolos de cada experimento (FERDINANDY et al., 2007).

\section{APLICAÇÃO EM EXPERIMENTOS}

Em experimentos com animais os danos do infarto e da isquemia podem ser executados por meio de estratégias manipuladas em pré-condicionamento remoto e pós-condicionamento. Nas estratégias pré-condicionadas, a isquemia é interrompida pela reperfusão, permitindo a avaliação da hipóxia, rápida localização do infarto, estresse térmico e vários fármacos receptores dependentes e independentes. No pré-condicionamento, desenvolvem-se eventos de isquemia com tempos pré-definidos, seguidos de reperfusão em outros órgãos. Tais estratégias são bastante utilizadas para avaliar danos nos tecidos endoteliais. Nas estratégias pós-condicionadas, as injúrias se tornam irreversíveis, os quadros de arritmia ventricular predominam. Estas ligações são mais utilizadas em protocolos envolvendo agentes farmacológicos (YANG, 2015).

Em humanos, infartos antroseptais por oclusão das artérias coronárias esquerda são raros, em torno de $13 \%$ do total. No entanto, estes procedimentos em pesquisa com animais são muito utilizados. Ao realizar a ligação após a primeira diagonal dos ramos septais, verificou-se $85 \%$ de infarto na parede livre do ventrículo direito, $100 \%$ da parede livre e ápice e $45 \%$ da massa do septo anterior do ventrículo esquerdo. Nesse caso, todos os animais vieram à óbito imediatamente após o procedimento (SAMSAMSHARIATA \& MOVAHEDB, 2015).

O fator de crescimento endotelial (EGF) é uma proteína importante usada na indução da angiogênese pós infarto agudo do miocárdo. Quando associado a "STSMLKA", uma proteína específica com função de interagir com a tropina cardíaca 
I, verificou-se a redução da cicatrização e melhora da função cardiaca em ambos os modelos experimentais, ratos e suínos (GARCIA et al., 2015). De forma semelhante, as proteínas do fator de crescimento endotelial vascular (VEGF), com 165 aminoácidos, foram injetadas intramiocárdica e equidistante da ligação da artéria ascendente esquerda. Após seis semanas, verificou-se a estimulação dos miócitos e a remodelação da matrix (JOKI et al., 2015).

O fator de crescimento de fibroblastos 21 apresenta uma importante função de modulador da insulina. No entanto, após o infarto, o efeito em remodelar é reduzido. Neste contexto, fator de crescimento de fibroblastos 21 foi administrado intramuscular em camundongos com dez semanas de vida. Após três dias da cirurgia de ligação da artéria coronária ascendente esquerda, houve uma disfunção sistólica e dilatação ventricular. Após duas semanas, diminuição dos miócitos apoptóticos, das citocinas pró-inflamação e aumento dos níveis plasmáticos das adiponectinas (SEDOVA et al., 2015).

Os antioxidantes são compostos químicos que reduzem as espécies reativas de oxigênio, diminuindo as arritmias cardiacas que ocorrem após as isquemias. O pigmento equinocromo do ouriço do mar A (2,3,5,7,8-pentahidroxil-6etil-1,4-naftaquinona) tem efeito cardioprotetor. Ao ser administrada em felinos, após a oclusão da artéria coronária ascendente esquerda, e o coração ser avaliado por períodos de 5, 10, 15 e 30 minutos de fibrilação, verificou-se não alterar a dispersão da repolarização das arritmias (CSEPANYI et al., 2015).

O betacaroteno é um composto químico proveniente da vitamina $A$, presente nas plantas. Quando administrado em ratos induzidos ao infarto, por meio da ligação da artéria coronária esquerda, pode apresentar efeito protetor ou agressor, em uma nova isquemia e reperfusão, ou contribue para desencadear as propriedades pro-oxidativas (KELLE et al., 2015).

A combinação do uso de rosuvastatina em isquemias pre-condicionantes e pós-condicionantes leva a uma diminuição da área de necrose e apresenta efeito cardioprotetor dos miócitos e células endoteliais (NETTER, 2015).

estabilidade dos mecanismos patológicos e fisiológicos em modelos animais experimentais, permite a reprodutibilidade dos efeitos e interações dos medicamentos com o procedimento de ligação da artéria coronária esquerda (XU et al., 2014).

\section{CONSIDERAÇÕES FINAIS}

As doenças cardiovasculares apresentam um elevado índice de mortalidade e hospitalização em todo o mundo. Podem se manifestar de maneira crônica, como a angina estável, ou de forma aguda, como a angina instável e o infarto agudo do miocárdio.

O infarto agudo do miocárdio pode ser uma das primeiras manifestações das doenças arteriais. Macroscopicamente, há presença de um trombo aderido as paredes das artérias, levando a diminuição ou obliteração da passagem total do fluxo sanguíneo, processo isquêmico. Este processo desencadeia diversas alterações celulares, como: diminuição do ATP, acúmulo de lactato, acidose e edema celular, levando a morte celular.

O alto índice de infarto e severas consequências nos humanos, levaram os pesquisadores a desenvolverem métodos de pesquisas em animais de uso experimental, que permitem simular doenças crônicas e agudas, testar procedimentos e medicamentos. Tais procedimentos também podem desobstruir as 
artérias e refinar as técnicas cirúrgicas de indução das doenças cardiovasculares. Dentre estas técnicas tem-se a ligação da artéria coronária esquerda.

A ligação da artéria coronária esquerda a 3 a $4 \mathrm{~mm}$ de sua origem, em animais de experimento, induz ao infarto agudo do miocárdio. Morfologicamente é possível avaliar a proliferação da circulação colateral, mensurar a área de necrose no coração, hipertrofia do ventrículo esquerdo e ativação das metaloproteases. Funcionalmente as alterações hemodinâmicas levam a diminuição do volume sistólico, débito cardíaco, pressão sistólica dentro do ventrículo esquerdo e aumento da pressão diastólica no final do ventrículo esquerdo. Clinicamente tem diminuição do débito cardíaco, lentidão de movimentos, alterações na pelagem, retardo de crescimento, peso corporal e dispnéia.

Mesmo diante das particularidades dos animais, os estudos de modelo de indução do infarto agudo do miocárdio, em modelos experimentais, são ideais para avaliar a intensidade das alterações fisiopatológicas que ocorrem em seres humanos.

\section{REFERÊNCIAS}

AGNOLETTI, G.; CARGNONI, A.; AGNOLETTI, L.; MARCELLO, M.; BALZARINI, P.; PASINI, E.; GITTI, G.; MARTINA, P.; ARDESI, R.; FERRARI, R. Experimental ischemic cardiomyopathy: insights into remodeling, Physiol Adap Hum Resp. Annals of Clinical \& Laboratory Science. v. 36, n. 3, p. 333-40, 2006. Disponivel em: http://www.annclinlabsci.org/content/36/3/333.short. Acesso em: 24 junho 2015.

BOPASSA, J. C. Protection of the ischemic myocardium during the reperfusion: between hope and reality. American Journal Cardiovascular Disease. v. 2, n. 3, p. 223-36, $2012 . \quad$ Disponivel em: http://www.ncbi.nlm.nih.gov/pmc/articles/PMC3427983/. Acesso em: 28 agosto 2015

BRASIL, Ministério da Saúde, Portal Brasil. Infarto. Disponível em: http://www.brasil.gov.br/saude/2012/04/infarto Antonio Cruz/ Infarto 17/04/2012. Acesso 18 setembro 2015.

BRASIL, Ministério da Saúde. Indicadores de mortalidade C.8 Taxa de mortalidade específica por doenças do aparelho circulatório Disponível em: http://tabnet.datasus.gov.br/cgi/tabcgi.exe?ldb2012/c08.def, Diretrizes 2014. Acesso em: 08 setembro 2015.

BRASIL, MS/SAS. Ministério da Saúde, Secretaria de Atenção à Saúde, Departamento de Atenção Básica. Cadernos de Atenção Básica: Prevenção clínica de doenças cardiovasculares, cerebrovasculares e renais. 14. Série A. Normas e Manuais Técnicos. p. 1-64, 2006. Disponível em: Bvsms.saude.gov.br/bvs/publicacoes/abcad14.pdf. Acesso em: 15 set 2015.

CHANG, J.; NAIR, V.; LUK, A.; BUTANY, J. Pathology of myocardial Infarction. Diagnostic Histopathology 19:1 Mini-Symposium: Cardiovascular Pathology II. p. 7-12, 2013. Disponível em: https://www.infona.pl/.../bwmeta1.element.elsevier-f. Acesso em: 18 setembro 2015.

COOPER, C. J.; PFEFFER, J. M.; FINN, P.; PFEFFER, M. A. Characteristics of a model of myocardial infarction produced by coronary artery ligation in the rat. 
Cardiovascular Pathology. v. 4, n. 3, p. 189-94,1995. Disponível em: http://www.ncbi.nlm.nih.gov/pubmed/25851007. Acesso em: 14 setembro 2015.

CSEPANYI, E.; CZOMPA, A.; ISTVAN, D. I.; EDINA, L.; BALLA, G.; BAK, I. Cardiovascular effects of low versus high-dose Beta-carotene in a rat model. Pharmacol Research journal. 2015. Disponivel em: doi: 10.1016/j. Acesso em: 23 maio 2015.

DÂNGELO, J. G.; FATTINI, C. A. Sistema Circulatório. In: Anatomia Humana Sistêmica e Segmentar. $2^{\circ}$ ed. Atheneu. São Paulo, 2000.

FERDINANDY, P.; SCHULZ, R.; BAXTER, G. Interaction of cardiovascular risk factors with myocardial ischemia/reperfusion injury, preconditioning, and postconditioning. Pharmcol Review. v. 59, n. 4, p. 418-58, 2007. Disponível em: http://www.ncbi.nlm.nih.gov/pubmed/18048761. Acesso em: 25 agosto 2015.

FERREIRA, C.; POVOA, R. Cardiologia para o Clinico Geral. Ed. 1. Atheneu. São Paulo, 1999.

GARCIA, L. F.; MATAVELI, F. D.; AMARAL, A. M.; MADER, A.; THEODORO, T. R.; JUSTO, G. Z.; PINHAL, M. A. S. Células envolvidas no remodelamento da matriz extracelular após infarto agudo do miocárdio. Einstein. v. 13, n. 1, p. 89-95, 2015. Disponível em: http://dx.doi.org/10.1590/S1679-45082015AO2970. Acesso em: 03 maio 2015.

GARTNER, L. P.; HIATT, J. Atlas de histologia. Ed 4. Guanabara. Rio de Janeiro,1993.

HOEHN, M.; ZHANGA, Y.; XUA, J.; GERGS, U.; BOKNIK, P.; WERDANA, K.; NEUMANNB. J.; EBELT H. Overexpression of protein phosphatase $2 A$ in a murine model of chronic myocardial infarction leads to increased adverse remodeling but restores the regulation of $\beta$-catenin by glycogen synthase kinase $3 \beta$. Inter. Journal of Cardiology. v. 183, p. 39-46, 2015.Disponível em: http://www.sciencedirect.com/science/article/pii/S016752731500114X. Acesso em: 14 outubro 2015.

HOUSER, S. R.; MARGULIES, K. M.; MURPHY, A. M.; SPINALE, F. G.; FRANCIS, G. S.; PRABHU, S. D.; ROCKMAN, H. Á.; KASS, D. A.; MOLKENTIN, J. D.; SUSSMAN, M. A.; KOCH, W. J. Animal models of heart failure. Circulation Research. v. 111, p. 131-150, 2012. Disponível em: doi: 10.1161/RES.0b13e3182582523. Acesso em: 26 junho 2015.

JOHNS, N.P.; OLSON, B.J. Experimental myocardial infarction. Annals of Surgery. p. 675-82, 1954. Disponivel em: http://www.ncbi.nlm.nih.gov/pubmed/13208115. Acesso em: 23 abril 2015.

JOKI, Y.; OHASHI, K.; YUASA, D.; SHIBATA, R.; ITO, M.; MATSUO, K. A.; KAMBARA, T.; UEMURA, Y.; HAYAKAWA, S.; HIRAMATSU-ITO, M.; KANEMURA, N.; OGAWA, H.; DAIDA, H.; MUROHARA, T.; OUCHI, N. FGF21 attenuates pathological myocardial remodeling following myocardial infarction through the adiponectin-dependent mechanism. Biochemical and Biophysical Research Communication. v. 459, p. 124-130, 2015. Disponível em: doi:10.1016/j.bbrc.2015.02.081. Acesso em: 18 julho 2015. 
JUNQUEIRA, L. C.; CARNEIRO, J. Histologia Básica. 8eed. Guanabara. Rio de Janeiro, 1995.

KELLE, H.; AKKOÇ, E.; UYAR, M.; ERDINÇ, O.; EVLIYAOGLU, S.; SARIBA, S.; TUNIK, C. The combined effect of rosuvastatin and ischemic pre- or postconditioning on myocardial ischemia-reperfusion injury in rat heart. European Review for Medical and Pharmacological Scicens. v. 19, p. 2468-76, 2015. Disponível em: http://www.ncbi.nlm.nih.gov/pubmed/26214784. Acesso em: 16 agosto 2015.

KLOCKE, R.; TIAN, W.; KUHLMANN, M. T.; NIKOL, S. Surgical animal models of heart failure related to coronary heart. Journal of Cardiovascular Disease Research. v. 74, p. 29-38, $2007 . \quad$ Disponivel em: DOI: http://dx.doi.org/10.1016/j.cardiores.2006.11.026. Acesso em: 13 maio 2015.

LOOI, J. et al. Morphology of congenital and acquired aortic valve disease by cardiovascular magnetic resonance imaging. European Journal Radiology. p. 1-11, 2015.

LUO, G.; LI, G.; ZHANG, X.; SHEN, L.; XIE, J.; ZHANG, J.; KITAKAZE, M.; HUANG, $X . ;$ LIAO, Y. Ablation of C/EBP homologous protein increases the acute phase mortality and doesn't attenuate cardiac remodeling in mice with myocardial infarction. Biochem and Biophys Res Commum. v. 464, p. 201-07, 2015. Disponivel em: doi: 10.1016/j.bbrc.2015.06.117. Acesso em: 17 setembro 2015.

MULDER, W.J.; JAFFER, F. A.; FAYAD, Z. A.; NAHRENDORF, M. Imaging and nanomedicine in inflammatory atherosclerosis. ScienceTranslationalMedicine. 2014. Disponível em: www.ScienceTranslationalMedicine.org. 2014;6:239. Acesso Agosto 2015.

NETTER, F. N. H. Atlas de Anatomia Humana. 6ed. Porto Alegre: Elsevier, 2015.

PESARO, A. E. P.; Serrano, C. V.; Nicolau, J. C. Infarto agudo do miocárdio Sindrome coronariana aguda com supradesnível do segmento ST. Revista Associação Medica Brasileira. v. 50, n. 2, p. 214-20, 2004. Disponível em: www.scielo.br/pdf/ramb/v50n2/20786.pdf. Acesso em: 24 outubro 2015.

RADILSON, C. G. MS, Portal Brasil. Doenças cardiovasculares causam quase $30 \%$ das mortes no País, 2011. Disponível em: http://www.brasil.gov.br/saude/2011/09/doencas-cardiovasculares-causam-quase30-das-mortes-no-pais. Acesso 18 setembro 2015.

SAMSAMSHARIATA, S. A.; MOVAHEDB, M.R. High rate of right ventricular infarction after ligation of mid left anterior descending artery in rats. Cardiovascular Revascularization Medicine. v. 6, p. 21-23, 2005. Disponivel em: doi:10.1016/j.carrev.2005.04.005. Acesso em: 29 setembro 2015.

SANTIAGO, M.B.; NASCIMENTO, A. M.; COUTO, W. C. S.; OLIVEIRA NETO, W. N.; LESSA, F. C. R.; FRANQUINI, J. V. M.; PINTO, V. D.; ANDRADE, T. U. Efeito da administração do Allium sativum sobre as alterações cardiovasculares de ratos Wistar com infarto do miocárdio. Revista de Ciência Farmácia Básica. v. 30, n. 1, p. 75-82. 2009. Disponível em: servbib.fcfar.unesp.br/seer/index.php/Cien_Farm/article/. Acesso em 26 agosto 2015. 
SEDOVA, K.; BERNIKOVA, O.; AZAROV, J.; SHMAKOV, D.; VITYAZEV, V.; KHARIN, S. Effects of echinochrome on ventricular repolarization in acute ischemia. Journal of Electrocardiology. v. 48, p. 181-86, 2015. Disponível em: doi: 10.1016/j.jelectrocard.2015.01.003. Acesso em: 24 agosto 2015.

SILVA, E. P.; SOUZA, J. L.; SILVA, T. N.; MARIANO, A. F. S.; SANTOS, D. L. A.; CUNHA, J. A. S.; PALMA, M. B. Comparação Anatômica entre o coração de humanos e aves. Recife. XIII Jornada de Ensino, Pesquisa e Extensão, 2013 Disponível em: www.eventosufrpe.com.br/2013/cd/resumos/R0630-3.pdf. Acesso 20 agosto 2015.

SIMÃO M., NOGUEIRA M S, HAYASHIDA M, CESARINO E J. Doenças cardiovasculares: perfil de trabalhadores do sexo masculino de uma destilaria do interior paulista. Revista Eletronica Enfermagem. v. 4, p. 27-35, 2002. Disponível em: http://dx.doi.org/10.5216/ree.v4i2.759. Acesso 20 agosto 2015.

SOLIMENE MC. Como diagnosticar e tratar a insuficiência coronária crônica. Grupo Editorial Moreira Jr. p.131-140. Disponível em: http://www.moreirajr.com.br/revistas.asp?fase=r003\&id_materia=2867. Acesso em: 20 set 2015.

SPOSITO, A.C., CARAMELLI, B.; FONSECA, F. A. H. Anatomia comparada do coração dos vertebrados: aspectos filogenéticos dos vertebrados e ontogenéticos da espécie humana. Sistema Anhanguera de Revistas Eletrônicas. p. 55-61, 2007. Disponível em: nead.uesc.br/arquivos/Biologia/.../anatomia-coracao-vertebrados.pdf. Acesso em: 20 setembro 2015.

TABTABAI, S.; YEH, D. F.; STEFANESCU, A.; KENNEDY, K.; YEH, R. W.; BHATT, A. B. National trends in hospitalizations for patients with single-ventricle anatomy. American Journal Cardiology. v. 116, p. 773-78, 2015. Disponivel em: doi: 10.1016/j.amjcard.2015.05.053. Acesso em: 17 junho 2015.

THYGESEN, K. T.; ALPERT, J. S.; JAFFE, A. S.; SIMOONS, M. L.; CHAITMAN, B. R.; HARVEY, D. Third Universal Definition of Myocardial Infarction Journal of the American College of Cardiology. v. 60, n.16, p. 1581-98, 2012. Disponivel em: doi: 10.1161/CIR.0b031e31826e1058. Acesso em: 18 agosto 2015.

VERDOUW, P. D.; DOEL, M. A. V.; ZEEUW, S.; DUNCKER, D. J. Animal models in the study of myocardial ischaemia and ischaemic Syndromes. Cardiovascular Research. v. 39, p. 121-35, 1998. Disponivel em: DOI: http://dx.doi.org/10.1016/S0008-6363(98)00069-8. Acesso em: 17 setembro 2015.

WHITE, H.; CHEW, D.P. Acute myocardial infarction. Thelancet. v.372, p. 570-584, 2008. Disponível em: www.thelancet.com. Acesso 15 setembro 2015

XU, Z.; ALLOUSH, J.; BECK, E.; WEISLEDER, N. A murine model of myocardial ischemia-reperfusion injury through ligation of the left anterior descending artery. Journal of Visualized Experiments. v. 3, p. 2-14, 2014. Disponivel em: doi: 10.3791/51329. Acesso em: 12 setembro 2015. 
YANG, Y. Modified VEGF targets the ischemic myocardium and promotes functional recovery after myocardial infarction. Journal of Control Release. v. 213, p. 27-35, 2015. Disponivel em: doi: 10.1016/j.jconrel.2015.06.036. Acesso em: 30 junho 2015.

ZORNOFF, L. A. M.; PAIVA, S. A. R.; MINICUCCI, M. F.; SPADARO, J. Infarto do Miocárdio Experimental em Ratos: Análise do Modelo. Arquivos Brasileiros de Cardiologia. v. 93, n. 3, p. 434-440,2009. Disponivel em: http://dx.doi.org/10.1590/S0066-782X2009001000018. Acesso em: 24 agosto 2015. 